\title{
LÍQUENS DE PIRAPUTANGA, MATO GROSSO DO SUL, BRASIL
}

\author{
Mariana Fleig' \\ Ivone Riquelme ${ }^{2}$
}

Recebido em 28-1-90. Aceito em 29-8-91.

RESUMO - Foram identificadas 72 espécies de líquens coletados em Piraputanga, município de Aquidauana; 23 taxa são registradas pela primeira vez para $o$ estado do Mato Grosso do Sul.

Palavras-chaves: Líquens, florística.

ABSTRACT - There are identified 72 species of lichens from Piraputanga, Aquidauana municipality; 23 taxa are reported for the first time for Mato Grosso do Sul State.

Key-words: Lichens, floristics.

\section{Introdução}

A vila Piraputanga, município de Aquidauana, está situada a oeste de Campo Grande, capital do Estado, $20^{\circ} 27^{\prime} \mathrm{S}, 55^{\circ} 29^{\prime} \mathrm{W}$, e alcança aproximadamente $200 \mathrm{~m}$ de altitude.

Segundo Russi (1975) o município de Aquidauana apresenta duas formações geológicas distintas: do Pantanal e Serrana; esta última composta por arenitos Paleozóicos de idade compreendida entre o Devoniano Inferior e o Carbonífero Superior, constitui regionalmente a série Aquidauana. Destacase o solo tipo $\mathrm{LE}_{7}$, mais conhecido como Latossolo Vermelho-escuro. A série Aquidauana é constituída essencialmente por sedimentos arenosos com cimento argiloso, conglomerados com cimento arenoso, apresentando camadas de siltito e folhelho.

Conforme Nimer (1979) Aquidauana (situada ao sul da Região Centro-Oeste do Brasil) apresenta clima subquente, úmido, com 3 meses de inverno seco; a temperatura média superior se situa em torno de $24^{\circ}-26^{\circ} \mathrm{C}$ e a média inferior, $18^{\circ} \mathrm{C}$. A precipitação anual é de $1.250 \mathrm{~mm}$, em regime tropi-

1 - Universidade Federal do Rio Grande do Sul, Deptº de Botânica. Av. Paulo Gama 110, Porto Alegre, 90.049 , RS.

2 - Depto de Química da Universidade Federal do Mato Grosso do Sul. Campo Grande, 79.100, MS. Bolsista Técnica do Projeto. 
cal, sendo $45-55 \%$ deste índice referente aos meses de novembro a janeiro.

Existem poucos registros da flora liquênica do Mato Grosso do Sul. Malme no fim do século passado percorreu Corumbá e imediações, seguindo para o norte em direção a Cuiabá, onde realizou maior número de coletas. Diversos trabalhos baseados nas coletas realizadas durante a Primeira Expedição Regnelliana, como Malme (1902, 1924, 1925, 1926, 1927, 1928, 1934 e 1936) e Redinger (1933 e 1935) são ainda os principais registros da flora liquênica local. Nas últimas décadas, as monografias sobre as parmeliáceas de Hale (1965, 1975 a e b, 1976 a e b), sobre o gênero Dirinaria, Awasthi (1975) e outras publicações baseadas em coletas mais recentes, como a série 'Lichenes Neotropicis' de Kalb (1981-84) e Brako et al. (1985), apresentam dados importantes para o estudo da flora liquênica regional.

O presente trabalho consiste na identificação taxonômica da flora liquênica da região, como contribuição ao projeto 'Estudo Químico de Líquens do Mato Grosso do Sul' desenvolvido pelo Departamento de Química da Universidade Federal do Mato Grosso do Sul e financiado pela Fundação Banco do Brasil.

\section{Material e métodos}

O ponto escolhido para as coletas fica próximo à vila Piraputanga, zona acidentada, com afloramentos rochosos, entre as escarpas rochosas da série Aquidauana e o rio do mesmo nome. A vegetação predominante é arbustiva a subarbustiva, escassa junto aos afloramentos rochosos em direção ao rio, um tanto densa e mais desenvolvida próximo ao paredão rochoso.

Foram realizadas coletas de líquens saxícolas, corticícolas e terrícolas na parte baixa com vegetação esparsa, na mata junto às escarpas rochosas e nas primeiras escarpas até aproximadamente $50 \mathrm{~m}$ de altura.

$\mathrm{O}$ material foi herborizado; parte se encontra nas dependências do Departamento de Química da Universidade Federal do Mato Grosso do Sul, parte no herbário do Departamento de Botânica da Universidade Federal do Rio Grande do Sul (ICN) para estudo. Algumas duplicatas foram enviadas a especialistas para confirmação das espécies.

A identificação dos taxa foi realizada junto ao Departamento de Botânica da UFRGS, consistindo em análises morfológicas e anatômicas do talo e frutificações, testes colorimétricos com hidróxido de potássio $(\mathrm{K})$, hipoclorito $(\mathrm{C})$, hidróxico de potássio mais hipoclorito $(\mathrm{KC})$, parafenileno $(\mathrm{P})$ e iodeto (I), conforme as necessidades de cada grupo, com base na literatura especializada. Algumas exsicatas foram submetidas a análises químicas como microcristalização e cromatografia de camada delgada, junto ao Departamento de Química da UFMS, sob a responsabilidade da prof ${ }^{\mathrm{a}}$ Neli Honda, coor- 
denadora do Projeto.

Teve-se a possibilidade de comparar algumas exsicatas com as coletadas por Malme - Exp. Regnell. I., Lich. - do Museu Nacional do Rio de Janeiro ( $\mathrm{R})$.

\section{Resultados e comentários}

O desconhecimento da flora liquênica local nos levou a listar um grande número de novas ocorrências; consideramos, no entanto, como já conhecidas para o Estado as espécies citadas para o Mato Grosso antes da sua divisão territorial.

Das espécies identificadas, 40 foram citadas anteriormente para o Mato Grosso, sendo mais de $50 \%$ destas relacionadas para Cuiabá ou arredores, e apenas 10 para áreas pertencentes ao Mato Grosso do Sul. Em alguns casos foram citadas espécies como tropicais, sem especificação do local de coleta ou ao menos a região; nestes casos teve-se por base a provável rota percorrida pelo coletor das exsicatas relacionadas como testemunhas, para admitir sua inclusão na flora conhecida do Centro-Oeste brasileiro. Todas as espécies já haviam sido citadas para o Brasil.

A maioria das espécies que identificamos são corticícolas, $69,5 \%$, sendo $21,7 \%$ saxícolas e indiferentes $8,6 \%$; também houve predominância das espécies de hábito foliáceo, 59,4\%, enquanto $39,1 \%$ são crustáceos ou escamosos.

As espécies são listadas em ordem alfabética, seguidas do nome e número do coletor e substrato. Eventualmente são tecidas considerações que julgamos oportunas. São assinaladas com asterisco as que constituem novidade para o Mato Grosso do Sul.

\section{Buellia curatellae Malme}

I. Riquelme 195A, 237. Córtex.

2. Buellia modesta (Kremplh.) Müll. Arg.

M. Fleig \& I. Riquelme 62. Córtex.

3. Buellia sulphurea Malme.

M. Fleig \& I. Riquelme 69, 88. Riquelme 191. Rocha.

4. Bulbothrix coronata (Fée) Hale

M. Fleig \& I. Riquelme 125. Córtex.

5. Bulbothrix fungicola (Lynge) Hale

M. Fleig \& I. Riquelme 82. Córtex.

* 6. Bulbothrix isidiza (Nyl.) Hale

I. Riquelme 33. Córtex.

Hale (1976b) cita-a para o Rio de Janeiro, São Paulo e Paraguai.

7. Bulbothrix suffixa (Stirt.) Hale. 
M. Fleig \& I. Riquelme 11, I. Riquelme 153. Córtex.

Hale (1976b) cita-a para as Américas Central e do Sul e Sul da África.

Brako et al. (1985) citam-na para a Serra do Cachimbo, região Centro-Oeste do Brasil.

8. Bulbothrix viridescens (Lynge) Hale

M. Fleig \& I. Riquelme 147. Córtex.

9. Calicium subcurtum Vain. var. denudatum Vain.

I. Riquelme 180, 195. Córtex.

10. Calopadia chacoënsis (Malme) Kalb \& Vêzda

M. Fleig \& I. Riquelme 144B, I. Riquelme 197. Córtex e sobre parmeliácea em rocha.

* 11. Caloplaca americana (Malme) Zahlbr.

M. Fleig \& I. Riquelme 35. Rocha.

Malme (1926) descreve-a para o Rio Grande do Sul e Argentina.

12. Caloplaca cinnabarina (Ach.) Zahlbr.

I. Riquelme 189. Rocha.

* 13. Caloplaca granularis (Müll. Arg.) C. Sambo

M. Fleig \& I. Riquelme 17. Córtex.

Malme (1926) cita-a para o Paraguai (Chaco, junto ao Rio Negro) e Bolívia. Osorio (1978) cita-a para o Rio Grande do Sul.

* 14. Caloplaca holocarpa (Hoffm.) Wade M. Fleig \& I. Riquelme 142. Rocha.

Malme (1926) cita-a como C. pyracea, para o Rio Grande do Sul.

15. Canoparmelia amazonica (Nyl.) Elix \& Hale

I. Riquelme 173. Córtex.

Hale (1976a) cita-a para o Mato Grosso, Minas Gerais e Rio de Janeiro.

16. Canoparmelia carneopruinata (Zahlbr.) Elix \& Hale

M. Fleig \& I. Riquelme 58. Córtex.

Hale (1976a) cita-a para Minas Gerais e São Paulo. Osorio (1977) cita-a para o Oeste do Paraná.

17. Canoparmelia caroliniana (Nyl.) Elix \& Hale

M. Fleig \& I. Riquelme 73, 76. Córtex.

Hale (1976a) cita-a para o Mato Grosso (Santana da Chapada). Nosso material apresenta grande parte do lado inferior claro.

* 18. Canoparmelia cryptochlorophaea (Hale) Elix \& Hale I. Riquelme 182. Córtex.

Hale (1976a) cita-a para o Ceará e Rio de Janeiro.

* 19. Canoparmelia texana (Tuck.) Elix \& Hale

M. Fleig \& I. Riquelme 72, 124. Córtex. 
Hale (1976a) cita-a para Minas Gerais, São Paulo, Santa Catarina e Rio Grande do Sul.

* 20. Chrysothrix candelaris (L.) Laundon

M. Fleig \& I. Riquelme 84. Córtex.

Laundon (1981) cita-a para o Sul do Brasil e observa que provavelmente seja cosmopolita.

21. Coccocarpia erythroxyli (Spreng.) Swinsc. \& Krog M. Fleig \& I. Riquelme 102, 122, I Riquelme 25. Córtex e rocha.

22. Coccocarpia palmicola (Spreng.) L. Arvidss. \& D. Gall. M. Fleig \& I. Riquelme 28, 106, I. Riquelme 26. Córtex e rocha.

23. Dermatocarpon australe Malme

I. Riquelme 185. Rocha.

Malme (1928) descreve-a para Corumbá. Comparamos o material com o 'Typus' (Exp. Regnell. I, Lich. s/n) do herbário R.

* 24. Dirinaria aegialita (Afz.) Moore

I. Riquelme 193. Córtex.

Awasthi (1975) cita-a para o Rio de Janeiro, Venezuela e outros países.

25. Dirinaria applanata (Fée) Awas.

I. Riquelme 71, 176. Córtex e rocha.

26. Dirinaria confluens (Fr.) Awas.

M. Fleig \& I. Riquelme 13, 19, 46, 112. Córtex.

27. Dirinaria confluens (Fr.) Awas. var. coccinea (Lynge) Awas.

M. Fleig \& I. Riquelme 117, I.Riquelme 31. Córtex.

28. Dirinaria melanocarpa (Müll. Arg.) Dodge

M. Fleig \& I. Riquelme 39, 74, 130, I. Riquelme 171. Córtex.

29. Glyphis cicatricosa (Ach.) Vain. f. confluens (Zenk.) Zahlbr.

M. Fleig \& I. Riquelme 99. Córtex.

Redinger (1933) cita-a para o Mato Grosso (Santana da Chapada), Paraguai e Rio Grande do Sul.

30. Graphis afzelii Ach.

M. Fleig \& I. Riquelme 65. Córtex.

* 31. Hciematomma puniceum (Ach.) Mass.

M. Fleig \& I. Riquelme 43, 105. Em córtex de Cereus.

Citada para o Brasil (Vainio, 1890). Nosso material apresenta talo rugoso e apotécio séssil.

32. Heterodermia microphylla (Kurok.) Skorepa

M. Fleig \& I. Riquelme 121. Rocha.

* 33. Heterodermia speciosa (Wulf.) Trevis.

I. Riquelme 172: Córtex.

Swinscow \& Krog (1988) citam-na como cosmopolita.

* 34. Hypotrachyna dactylifera (Vain.) Hale

M: Fleig \& I. Riquelme 107, 126. Córtex. 
Citada para o Rio de Janeiro e São Paulo (Hale, 1975a).

* 35. Hypotrachyna livida (Tayl.) Hale

M. Fleig \& I. Riquelme 24. Córtex.

Hale (1975a) cita-a para o Leste e Sul do Brasil.

36. Hypotrachyna palmarum (Lynge) Hale

M. Fleig \& I. Riquelme 123. Rocha.

37. Laurera sanguinaria Malme

M. Fleig \& I. Riquelme 68, I. Riquelme 169. Córtex.

38. Lecanora caesiorubella Ach. subsp. glaucomodes (Nyl.) Imsh. \& Brodo

M. Fleig \& I. Riquelme 22, 97, I. Riquelme 36. Córtex.

Imshaug \& Brodo (1966) citam-na para o Mato Grosso (Serra da Chapada).

39. Lecanora oreinodes (Koerb.) Hertel \& Rambold

M. Fleig \& I. Riquelme 30. Rocha.

40. Lecidea russula Ach.

M. Fleig \& I. Riquelme 15, 83, 96, 131, Riquelme 30. Córtex.

* 41. Myriotrema wightii (Tayl.), Hale

I. Riquelme 67, 73, 160. Córtex e rocha.

Osorio \& Fleig. (1987) citam-na para o Rio Grande do Sul.

* 42. Ochrolechia subpallescens Vers.

I. Riquelme 35. Córtex.

Verseghy (1962) cita-a para o Uruguai e Argentina. Osorio \& Fleig (1983) citam-na para o Rio Grande do Sul.

43. Parmeliella pannosa (Sw.) Müll. Arg.

I. Riquelme 69. Rocha.

Malme (1925) cita-a para o Mato Grosso (Serra da Chapada).

44. Parmotrema argentinum (Kremplh.) Hale

M. Fleig \& I. Riquelme 98. Córtex.

45. Parmotrema cornuta (Lynge) Hale

I. Riquelme 19. Córtex.

* 46. Parmotrema crassescens (Stirt.) Hale

I. Riquelme 199. Córtex.

* 47. Parmotrema delicatulum (Vain.) Hale

M. Fleig \& I. Riquelme 05, 60, 127. Rocha.

Hale (1965) cita-a para Minas Gerais, Paraná e Uruguai.

48. Parmotrema dilatatum (Vain). Hale

M. Fleig \& I. Riquelme 41, 51; 61, L.Riquelme 20. Córtex.

* 49. Parmotrema flavescens (Kremplh.) Hale

M. Fleig \& I. Riquelme 143. Rocha.

Hale (1965) cita-a para o Rio de Janeiro.

50. Parmotrema latissimum (Fée) Hale

M. Fleig \& I. Riquelme 57. Córtex. 
51. Parmotrema mesotropum (Müll. Arg.) Hale

I. Riquelme 190. Córtex.

* 52. Parmotrema praesorediosum (Nyl.) Hale.

M. Fleig \& I. Riquelme 42, 63. Córtex.

Hale (1965) cita-a para o Rio de Janeiro, Minas Gerais, Rio Grande do Sul, Paraguai e Argentina.

53. Parmotrema tinctorum (Nyl.) Hale

M. Fleig \& I. Riquelme 16, I. Riquelme 23. Córtex.

* 54. Peltula tortuosa (Nees) Wetm.

M. Fleig \& I. Riquelme 34, I. Riquelme 163A. Rocha.

Vainio (1890) cita-a para o Rio de Janeiro.

55. Pertusaria commutata Müll. Arg.

I. Riquelme 161. Córtex.

Malme (1936) cita-a para o Mato Grosso (Santana da Chapada),

Rio Grande do Sul e Paraguai.

56. Pseudoparmelia sphaerospora (Nyl.) Hale

M. Fleig \& I. Riquelme 38, I. Riquelme 156, 170. Córtex.

Hale (1976a) cita-a para o Mato Grosso. O material logo após coletado não apresenta a reação de coloração citada na literatura (Hale, 1976a), o lado superior do talo pode ser esverdeado ou amarelado.

57. Pyxine berteriana (Fée) Imsh.

I. Riquelme 201. Córtex.

Kalb (1987) cita-a para Campo Grande; as demais espécies do gênero são também citadas por Kalb para o Mato Grosso do Sul.

58. Pyxine coccifera (Fée) Nyl.

M. Fleig \& I. Riquelme 78. Córtex.

59. Pyxine coralligera Malme.

M. Fleig \& I. Riquelme 79. Córtex.

60. Pyxine daedalea Krog \& R. Sant.

M. Fleig \& I. Riquelme 44, 52, 54, 108, I. Riquelme 181; 196. Córtex.

61. Pyxine obscurascens Malme

M. Fleig \& I. Riquelme 109, 146. Córtex.

62. Pyxine pungens Zarlbr.

M. Fleig \& I. Riquelme 104, 110; 115, 132, I. Riquelme 196A. Córtex.

63. Ramalina continentalis Malme

I. Riquelme 34. Rocha.

Malme (1934) descreve-a para o Mato Grosso (próximo a Cuiabá, Serra da Chapada) e Paraguai.

64. Relicina abstrusa (Vain.) Hale

M. Fleig \& I. Riquelme 80, 101: Córtex e rocha. 
Hale (1975b) cita-a para o Mato Grosso, Minas Gerais, Rio de Janeiro e Rio Grande do Sul.

65. Rinodina deminuta Malme

M. Fleig \& I. Riquelme 04. Rocha.

Malme (1902) cita-a para o Rio Grande do Sul e Mato Grosso (Cuiabá).

66. Rinodina mülleri Malme

M. Fleig \& I. Riquelme 29, I. Riquelme 75A. Rocha.

67. Sarcographa medusulina (Nyl.) Redgr.

M. Fleig \& I. Riquelme 100. Córtex.

Redinger (1933) inclui a espécie na chave analítica, citando-a para

o Brasil. Müller (1887) descreve-a.

* 68. Trapelia coarctata (Turn.) M. Choisy

M. Fleig \& I. Riquelme 134B. Rocha.

Hertel (1970) cita-a para o Sudeste do Brasil.

69. Trypethelium aeneum (Eschw.) Zahlbr.

M. Fleig \& I. Riquelme 10, 55. Córtex.

70. Trypethelium eluteriae Sprengel

I. Riquelme 178. Córtex.

Malme (1924) cita-a para o Mato Grosso (Corumbá).

71. Trypethelium subcatervarium Malme

M. Fleig \& I. Riquelme 66. Córtex.

* 72. Xanthoparmelia succedans Elix \& Johnston

M. Fleig \& I. Riquelme 02, 09, 32, 33, 64, I. Riquelme 184. Rocha.

Elix et al. (1986) citam-na para o Brasil, zonas costeiras e zonas áridas.

\section{Referências bibliográficas}

AWASTHI, D.D. 1975. A monograph of the lichen genus Dirinaria. Bibliotheca Lichenologica v. 2, J. Cramer, Vaduz.

BRAKO, L., M.J. DIBBEN \& I. AMARAI 1985. Preliminary notes on the macrolichen of Serra do Cachimbo, Northcentral Brazil. In: Contribuições do Projeto Flora Amazonica, pt. 2 Acta Amazonica suplemento 15 (1-2): 123-35.

ELIX, J. A., J. JOHNSTON, \& P.M. ARMSTRONG, 1986. A revision of the lichen genus Xanthoparmelia in Australia. Bull. Br. Mus. Nat. Hist. 15(3): 163-362.

HALE, M. E. 1965. A monograph of Parmelia subgenus Amphigymnia. Contr. U.S. Nat. Herb. 36(5): 193-358. -1975a. A revision of the lichen genus Hypotrachyna (Parmelia- 
ceae) in Tropical America. Smith. Contr. Bot. 25:1-73.

.1975b. A monograph of the lichen genus Relicina (Parmelia-

ceae). Smith. Contr. Bot. 26:1-32.

1976a. A monograph of the lichen genus Pseudoparmelia Lynge (Parmeliaceae). Smith. Contr. Bot. 31:1-62.

1976b. A monograph of the lichen genus Bulbothrix Hale (Parmeliaceae). Smith. Contr. Bot. 32:1-29.

HERTEL, H. 1970. Trapeliaceae-eine neue Flechtenfamilie. Ber. Dtsch. Bot. Ges. N.F.,4: 171-85.

IMSHAUG, H.A. \& I.M. BRODO, 1966. Biosystematic studies on Lecanora pallida and some related in the Americas. Nova Hedwigia 12(1-2): 1-59.

KALB, K. 1981-1984. Lichenes Neotropici. fasc. 1-8 n-1.350, Neumar$\mathrm{kt} / \mathrm{Opf}$. theca Lichenologica v. 24, J. Cramer, Berlin.

LAUNDON, J.R. 1981. The species of Chrysothrix. Lichenologist 13(2):101-21.

MALME, G.O.A. 1902. Die Flechten der ersten Regnellschen Expedition. II. Die Gattung Rinodina (Ach.) Stiz. Bih. K. Svenska Vetensk Akad. Handl. 28(3):1-53.

. 1924. Die Flechten der ersten Regnellschen Expedition. Astrotheliaceae, Paratheliaceae und Trypetheliaceae. Ark. Bot. 19(1): 1-34. . 1925. Die Pannariazeen des Regnellschen Herbars. Ark. Bot. 20A(3): 1-23.

1926. Lichenes blasteniospori Herbarii Regnelliani. Ark. Bot. 2OA(9):1-51.

1928. Lichenes pyrenocarpi aliquot in Herbario Regnelliano asservati. Ark. Bot. 22A(6):1-11.

1934. Die Ramalinen der ersten Regnellschen Expedition. Ark. Bot. 26A(12):1-9.

1936. Pertusariae Expeditionis Regnellianae primae. Ark. Bot. 28A(9):1-27.

MULLER, J. (Argoviensis). 1887. Lichenologische Beiträge XXV. Flora, Regensburg no 1087.

NIMER, E. 1979. Climatologia do Brasil. Série: Recursos Naturais e Meio Ambiente no 4. IBGE. Rio de Janeiro.

OSÓRIO, H.S. 1977. Contribution to the lichen flora of Brazil III. Lichens from Western Parana. Acta Biol. Par. 6(1-4):3-7.

1978. Contribution to the lichen flora of Brazil IV. Lichens from Southern Rio Grande do Sul. Bryologist 81(3):452-54. 
\& FLEIG, M. 1983. Contribution to the lichen flora of Brazil XI. Lichens from Santa Maria, Rio Grande do Sul State. Phytologia, 53(2): 138-40.

. \& FLEIG, M. 1987. Contribution to the lichen flora of Brazil XIX. New or additional records from Santa Maria, Rio Grande do Sul State. Comunicaciones Botanicas del Museo de Historia Natural de Montevideo 5(81): 1-8.

REDINGER, K. 1933. Die Graphidineen der ersten Regnellschen Expedition nach Brasilien 1892-94. I. Glyphis, Medusulina und Sarcographa. Ark. Bot. 25A(13):1-20.

. 1935. Die Graphidineen der ersten Regnellschen Expedition nach Brasilien 1892-94. Graphis und Phaeographis, nebst einem Nachtrage zu Graphina. Ark. Bot. 27A(3):1-103.

RUSSI, G.C. 1975. Subsídios para o estudo de uma região: Aquidauana. (Polígrafo de divulgação interna, Centro Universitário de Aquidauana, Universidade Federal do Mato Grosso do Sul).

SWINSCOW, T.D.V. \& H. KROG. 1988. Macrolichens of East Africa. British Museum (Natural History). London.

VAINIO, E.A. 1890. Etude sur la classification naturelle et la morphologie des lichens du Brésil. Acta Soc. Fauna Flora Fenn. 7(1):1-247.

VERSEGHY, K. 1962. Die Gattung Ochrolechia. Nova Hedwigia Beih. 1. 